\title{
Maxwell's Fishpond
}

\author{
Paul Kinsler. Jiajun Tan, Timothy C. Y. Thio, Claire Trant, and Navin Kandapper \\ Department of Physics, Imperial College London, \\ Prince Consort Road, London SW7 2AZ, United Kingdom.
}

(Dated: Thursday $28^{\text {th }}$ June, 2018)

\begin{abstract}
Most of us will have at some time thrown a pebble into water, and watched the ripples spread outwards and fade away. But now there is also a way to reverse the process, and make those ripples turn around and reconverge again, ... and again, and again. To do this we have designed the Maxwell's Fishpond, a water wave or "Transformation Aquatics" version of the Maxwell's fisheye lens [1. 2]. These are transformation devices where wave propagation on the surface of a sphere is modelled using a flat device with spatially varying properties. And just as for rays from a point source on a sphere, a wave disturbance in a Maxwell's fisheye or Fishpond spreads out at first, but then reforms itself at its opposite (or complementary) point. Here we show how such a device can be made for water waves, partly in friendly competition with comparable electromagnetic devices [3] and partly as an accessible and fun demonstration of the power of transformation mechanics. To the eye, our Maxwell's Fishpond was capable of reforming a disturbance up to five times, although such a feat required taking considerable care, close observation, and a little luck.
\end{abstract}

What can you see in the video at http://www.qols.ph.ic.ac.uk/ kinsle/files/MFishpond/ ?

This is an updated preprint of the published article ${ }^{1}$ Kinsler et al., Eur. J. Phys. 33, 1737 (2012). http://iopscience.iop.org/0143-0807/33/6/1737

\section{INTRODUCTION}

Transformation Optics 任 and Transformation Acoustics $[5$ are powerful new techniques used to design transformation devices, which usually tend to the exotic - invisibility or event cloaks [6, illusion generators, and so on. Unfortunately, none are useful as demonstration devices accessible to non-specialists.

Here, in contrast to this typical situation, we describe how to make a transformation device (T-device) that fits on a tabletop and which controls water waves visible to the naked eye. Although T-devices such as cloaks cannot be made with simple isotropic materials, one important type can: one whose design principle transforms from waves travelling on the surface of a uniform sphere, to waves travelling on a flat disk. The transformation used preserves the properties of the original wave propagation - that of circular trajectories of equal circumference - by making the disk properties non-uniform: here, we make a shallow pond with varying depth.

In optics, such a device is known as the Maxwell's fisheye lens [1, 2]. It has a long history, originally being

\footnotetext{
*Electronic address: Dr.Paul.Kinsler@physics.org

${ }^{1}$ Statement required by the publisher of the EJP: This is an author-created, un-copyedited version of an article accepted for publication in the European Journal of Physics. IOP Publishing Ltd is not responsible for any errors or omissions in this version of the manuscript or any version derived from it. The definitive publisher-authenticated version is available online at doi:10.1088/0143-0807/33/6/1737.
}

proposed in a problem set in the 1850's [7. 8]. Until recently an obscure theoretical curiosity, the device was brought to wider attention by controversial claims that such a device could generate perfect optical images $[9$. Leaving aside that debate (see 10 for a recent critical summary), Leonhardt usefully noted that actually building the device becomes possible if you take only the central portion and surround it by a mirror [9]. This trick also makes the water wave version easier to build. But what makes a Maxwell's fisheye lens - or its Fishpond counterpart - interesting?

As in an ordinary pond, a pointlike wave source anywhere on the surface of a Maxwell's Fishpond generates an outgoing set of ripples. However, in a Maxwell's Fishpond, the ripples do not just spread out and disperse, they also converge on the opposite side, before again diverging and travelling back to the start where they again reconverge, and repeat this process until they eventually dissipate. This is just as light does in the optical Maxwell's fisheye lens [11, and is exactly what rays or waves confined on the surface of a sphere would do. More generally, the Maxwell's fisheye is one of a more general class of classical transformation optics devices 近; and these others, such as the Eaton or Luneberg lenses, will also have accessible transformation aquatics equivalents based on water waves - just as they do in the more technologically exotic field of plasmonics [12, 13].

Here we will derive the water depth profile needed to make a Maxwell's Fishpond in the shallow water limit, and compare that profile to a simple approximation using a shallow spherical dome. Although the approximation can work surprisingly well, our accurate device does better, and can hint at - at least to the eye - up to five successive refocussings! More rigorously, we also present simulation results indicating how the device works in practise, as well as two experimental schemes set up with relatively little demands on equipment. 
Contributions to this work were as follows: PK conceived the Maxwell's Fishpond idea, and built the first crude prototype. He also designed the version used here, but with students NK and TT shadowing that design process. NK and TT did the first experiments, and CT and JT followed next; all four writing reports and giving presentations as part of their coursework. PK was the primary author of this paper, assisted by material from the student reports, he also did all the computer simulation work. CT, JT, and TT also assisted in the preparation of this final manuscript.

\section{FISHEYE, FISHPOND}

The Maxwell's fisheye concept is based on mimicking the properties of ray trajectories on the surface of a sphere using a flat surface with spatially modulated properties. This is interesting, because on a sphere any set of rays emitted from a point follow their individual "great circle" geodesics, and so will automatically converge on the exact opposite side of the sphere. Thus, any flat T-device version should also have this property - rays diverging from any point would automatically focus at the complementary point of the plane. Thus, both on the sphere and in the fisheye, an object at any point is guaranteed to form an image; this is most certainly not the case in ordinary imaging systems. Further, the rays would then re-diverge before converging again; in an ideal ray device, these image reformations would continue forever.

To achieve the transformation from a spherical device with its curved surface, to a flat one, we use a stereographic projection. Imagine a sphere sitting with its south pole on a flat sheet, as shown on the upper part of fig. 11. Then any point (e.g. $A$ or $B$ ) on the sphere is mapped onto the sheet by following a straight line from the north pole, through $A$ (or $B$ ), and onward until it intersects the sheet at $A^{\prime}$ (or $B^{\prime}$ ). In this way the curved southern hemisphere maps onto a disk on the flat sheet centered on the south pole. The northern hemisphere is mapped to points further away; with points very near the north pole being extremely remote, and the north pole itself having to be omitted.

Of course, although we would like to make a fisheye (or Fishpond) based on this projection, we do not want one that is infinitely big. We therefore follow Leonhardt [9] and place a mirror at the equator, confining all ray paths to the southern hemisphere, and so confining all projected rays inside a circle with twice the sphere's radius. Since both hemispheres have the same properties, the ray properties are preserved - although the great circles are now folded back on themselves and have a kink where they are reflected, they still are guaranteed to form a image of any point.

The process of opening out and flattening the surface of a sphere into an equivalent sheet, as if it were a map projection used for an atlas, has an important feature.

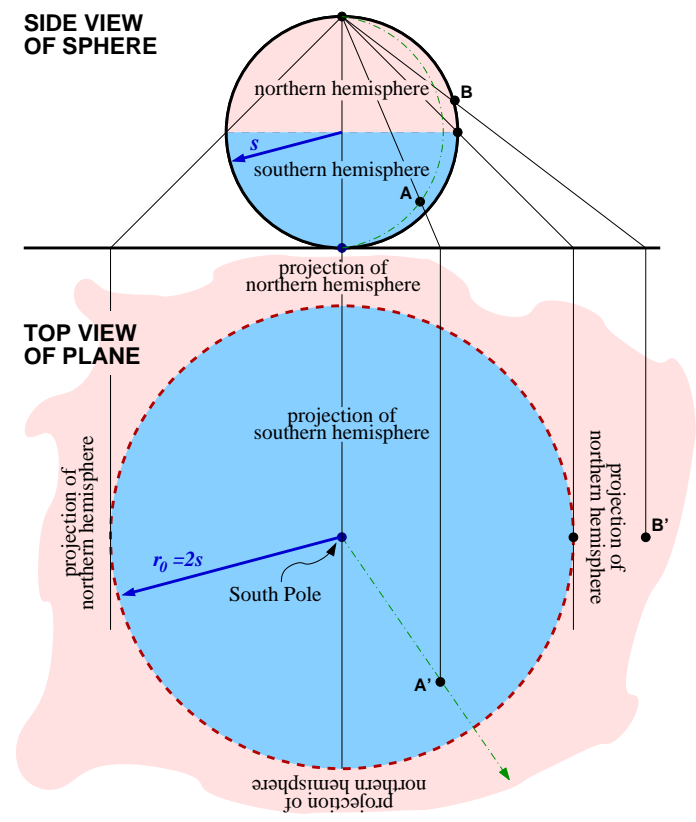

FIG. 1: The sphere-to-plane fisheye projection can be imagined by considering a transparent sphere with a light source placed at the north pole $N$, objects on the sphere then cast a shadow on the plane matching the projection. The southern hemisphere of the sphere of radius $s$ becomes a finite disk of radius $r_{0}=2 s$, whereas the northern hemisphere becomes the entire plane that remains outside the disk. Lines from pole-to-pole (meridians, or lines of longitude) on the sphere become radial lines (see e.g. the dot-dashed line), circles on the sphere parallel to the equator (parallels, or circles of latitude) become projected circles whose size depends on how far north or south of the equator (dashed line) they are.

Regions near the equator are stretched and expanded, while those near the south pole are only slightly changed. Mathematically we can define a complex quantity $z=$ $x+\imath y$, where $x, y$ represent the Cartesian coordinate on the plane. This means that any point $(X, Y, Z)$ (or at angles $\theta, \phi)$ on the unit sphere is projected (or "mapped") down onto

$$
z=\frac{X+\imath Y}{1-Z} \quad=\exp (\imath \phi) \cot (\theta / 2) .
$$

This means that a given line element $d S^{2}=d X^{2}+d Y^{2}+$ $d Z^{2}$ on the sphere is transformed into a line element in the plane $d R^{2}=d x^{2}+d y^{2}$ that progressively lengthens as we move towards the equatorial perimeter at $r=1$.

$$
\frac{2}{1+r^{2}} d R^{2}=d S^{2}
$$

where $r^{2}=x^{2}+y^{2}$, and we can also note that angles on the sphere are preserved when projected onto the plane.

This length transformation means that an object (or ray) travelling at a fixed speed on the sphere will have a projection on the plane that travels faster the closer it gets to the north pole. Thus a fixed object speed $v_{0}$ on the sphere is projected onto the disk as a radially varying 
velocity profile $v(r)$. Because of the way the projection works - or the line element $d S$ converts to $d R$ - the velocity profile $v(r)$ for a sphere of radius $r_{0} / 2$ is projected onto its counterpart disk of radius $r_{0}$ as

$$
v(r)=v_{0}\left[1+\left(\frac{r}{r_{0}}\right)^{2}\right] .
$$

Any disk which transports objects or waves with this velocity profile will be a T-device representing a sphere. Note that this velocity profile has a counterpart in a gradient-index version of Snell's law that steers propagating rays so that they match the paths that follow from projections of the great circle paths on the sphere. Indeed, from a mathematical perspective we might have expected this, because the projection preserves angles, which also means that the device can be made with isotropic materials.

\section{A. Optics}

In optics, one can actually make a thin spherical shell that will guide light inside it (see e.g. [14), just like the spherical reference device for a planar fisheye lens. But to obtain a design for the flat Maxwell's fisheye lens we need to design an optical device that has the light speed profile defined in eqn. (3) by modulating the refractive index. If starting with a shell with refractive index $n_{0}$ (and hence speed of light $c^{\prime}=c / n_{0}$ ), we can convert this to a disk with a radially varying refractive index profile

$$
n(r)=\frac{n_{0}}{1+\left(r / r_{0}\right)^{2}},
$$

where $n_{0}$ is the maximum refractive index we can achieve, and $r_{0}$ is our desired radius scale. In the original fisheye lens, $r$ was unbounded, causing the device to need unrealistically small values of $n$ at large radii $r$. The introduction of an equatorial mirror, as discussed above, circumvents this restriction; and for $n_{0} \geq 2$ the minimum refractive index required is always $\geq 1$. Electromagnetic Maxwell's fisheye lenses have been made (e.g. [3, 15 [17]), but require significant technological skill to build and investigate. Hence our interest in water waves, which gives a much wider audience access to these interesting devices.

\section{B. Water waves}

We want to make a Fishpond, not a fisheye; water waves are easily visible, intuitive, low-tech, and are accessible and safe for a wide variety of ordinary people. Nevertheless, experimental water wave systems can still be used as models for quite a surprising variety of phenomena: e.g. event horizons and Hawking radiation [18, 19] and neutron star collapse 20 .

To obtain a design for such a Maxwell's Fishpond we need only work out how to design a device that has the speed profile defined in eqn. (3). In general, water waves can have a complicated and nonlinear behaviour, so constructing a general fishpond will be either very difficult or impossible. But there is an important subset of water waves for which we can get a simple solution - those waves that occur in very shallow water.

For water of a constant depth that is significantly less than a wavelength, the wave speed for small waves is simply 21

$$
v_{w}=\sqrt{g d}
$$

where $g=9.81 \mathrm{~m} / \mathrm{s}^{2}$ is the gravitational acceleration and $\mathrm{d}$ is the water depth. In this extreme limit, other factors such as the wave amplitude and wavelength no longer matter, and we can control any suitable wave with the same depth modulation. Further, as long as $d(r)$ varies slowly over wavelength scales, we can use the formula to describe waves travelling across a varying depth profile $d(r)$.

If the centre of the fishpond has depth $d_{0}$, the water wave speed there is $v_{w}(0)=\sqrt{g d_{0}}$. Thus the radial wave velocity profile will be

$$
v_{w}(r)=v_{w}(0) \sqrt{\bar{d}},
$$

where $\bar{d}(r)=d(r) / d_{0}$ is the relative depth profile.

Thus, comparing eqns. (3) and (6) we see that to match the two velocity profiles we need to have

$$
\begin{aligned}
\sqrt{\bar{d}(r)} & =\left[1+\left(r / r_{0}\right)^{2}\right] \\
\bar{d}(r) & =\left[1+\left(r / r_{0}\right)^{2}\right]^{2} \\
& =1+2\left(r / r_{0}\right)^{2}+\left(r / r_{0}\right)^{4} .
\end{aligned}
$$

The parameters we chose for our Fishpond were based on an assumed water wavelength of about $20 \mathrm{~mm}$; the result can be seen in fig. 2.

One might also construct other types of geodesic lens 11, 2, 22 using water waves, or other types of acoustic waves 23., as discussed in the appendix at VI A and VIB.

\section{MODELLING}

We tested our design using computer simulations for a variety of cases ranging from those applicable to the ideal Maxwell's Fishpond and approximate Fishponds, to a full finite element simulation for our Fishpond device. For the idealized comparisons, we used the fact that the fisheye and Fishpond behave in an essentially identical manner, once the distinctions between the polarizable EM field and scalar water waves have been accounted for. This means that since an ideal Fishpond has the same properties as an ideal fisheye lens, FDTD simulations of Maxwell's equations for the fisheye lens will indicate the behaviour of an ideal Fishpond. 




FIG. 2: The Maxwell's Fishpond. (a) A cross section with the vertical scale grossly exaggerated for clarity. The indicated dimensions are those used for our actual device, but heights or widths can be rescaled freely - subject to the proviso that ripples will have a wavelength longer than the maximum depth. In fact, an even shallower Fishpond would provide a better match to this criterion, but since water has a significant surface tension, this makes covering the centre region problematic. (b) A photograph of our device.

To get an initial estimate of the importance of the correct depth profile, we used MEEP 24] FDTD simulations of Maxwell's equations. This approach was taken because we already had such EM simulations running, and because the MEEP software is flexible, open source, and freely available. All that is required is to converted our chosen depth profiles back into a a refractive index profile using the reverse of the process that led to eqn. (9). Sample MEEP control files are available in appendix VID. As well as the exact Maxwell's Fishpond, and amongst other variations, we modelled an approximate depth profile based on a shallow spherical cap (SC). It turns out that as long as the correct 1:4 ratio of minimum to maximum depths is maintained, this worked remarkably well.

In fig. 3 we can see the simulation results equivalent to our shallow water wave model, showing snapshot pairs that demonstrate the image reformation properties. We see that the ideal Maxwell's fisheye lens and Fishpond will give accurate refocussing (fig. $3(a, b)$ ), and this is repeated very many times before the performance starts to degrade due to the dispersion caused by how different wavelengths interact with the finite-sized geometry. (see e.g. [11]). Next, the simulations matching the approximate domed pond profile, do quite well (see fig. $3(c, d)$ ), but with some distortion clearly evident on the second reformation. However, the chosen "best reformation" snapshots of the domed pond flatter slightly, as the frames before and after shown a significant ellipticity; and the third reformation (not shown), whilst still giving a localised wave bunch, has lost its concentricring character. Finally, simulations of a flat-bottomed pond (see fig. 3(e,f)) show only a poor attempt at a first
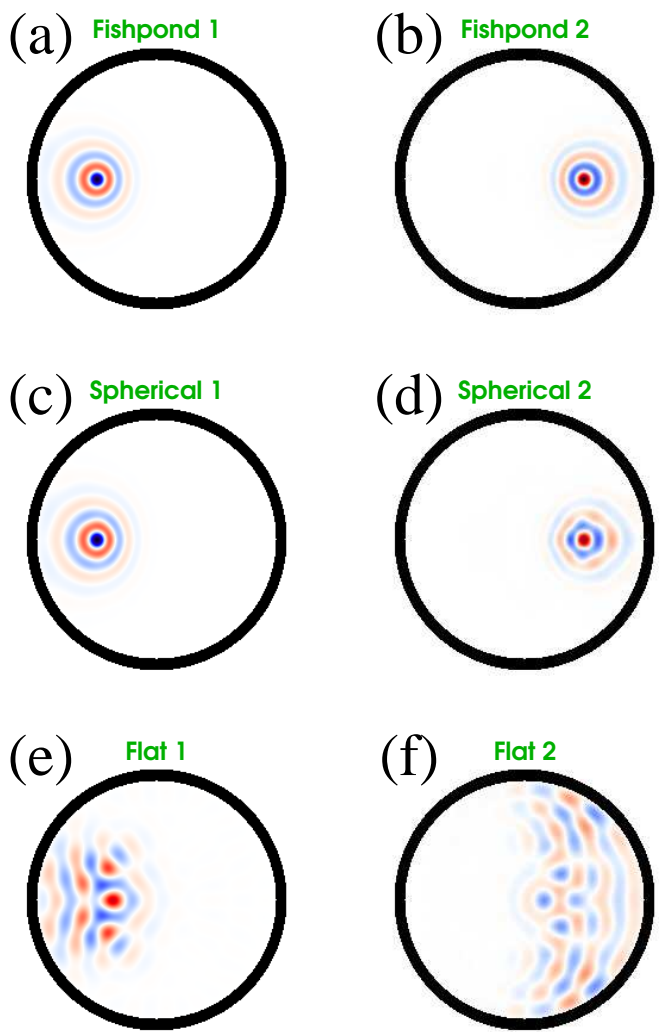

FIG. 3: Snapshots from simulations representing an ideal Maxwell's Fishpond (a,b), an approximate Fishpond with a spherical-cap (SC) depth profile (c,d), and an ordinary flatbottomed fishpond $(e, f)$. The upper frames $(a, b)$ show the fisheye/pond wave patterns near the first and second refocussing times; the middle ones $(\mathrm{c}, \mathrm{d})$ the approximate $\mathrm{SC}$ pond, and the lower two $(\mathrm{e}, \mathrm{f})$ show the non-focussing behaviour of a flat-bottomed pond when the ripples are not started at the exact centre. To the eye, the second Fishpond reformation (b) is essentially identical to the original source wave.

focus, followed by a rapid evolution towards an apparently random pattern with no reformations apparent at all. Other simulations including a variety of strengths of non-radial distortion of the Fishpond depth profile were also performed. Depth variations of about $10 \%$ away from the exact profile do give tolerable results for the first few image reformations, but the distortion strongly degrades the beautiful concentric-ring character of the exact Maxwell's Fishpond.

Unfortunately, it is hard to build a real water wave device that works perfectly in the shallow water limit. Most notably, we will expect to see some residual dispersion, due to the depth-dependent speeds of different wavelength ripples and the effects of surface tension; this is discussed later in section $\mathrm{V}$. Thus the brief reformations expected in the ideal case will blur out into longer process as different wave components refocus at different times, and for the shorter $\lambda$ waves, the depth profile will 


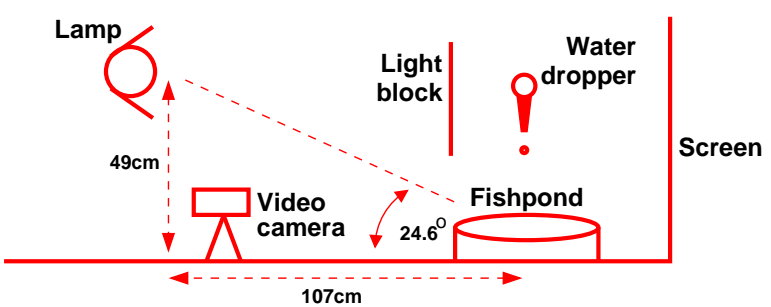

FIG. 4: The experimental setup of NK and TT. The water dropper was held in place by a retort stand (not shown).

be less perfect.

More realistic finite element simulations were also done using the open source simulator OpenFOAM 25 with the interMixingFoam engine on a fast desktop PC. Despite computational constraints, the simulations gave good results, with the effect of dispersion demonstrated, as can be seen in the appendix at VIC.

\section{EXPERIMENTS}

To make the Maxwell's Fishpond, our departmental Mechanical Workshop machined us a brass insert with the necessary depth profile (see fig. 2) and mounted this in a nylon ring, with a small notch to indicate the preferred water level. The choice of brass and nylon was based on convenience, not necessity, any waterproof materials could suffice. Our brass insert was not perfectly smooth, but was machined to tolerances of much less than $1 \mathrm{~mm}$.

Two schemes for obtaining quantitative data were used: one by NK and TT, the other by CT and JT. Both were influenced by the fact that although viewing the Fishpond directly gives a very strong impression of how well it works, this human perception does not translate easily into objective experimental data. Although even tiny ripples were surprisingly visible to the eye when in motion, it was less easy to get good experimental images.

Being the first attempt, the NK/TT experimental setup was relatively simple. In a darkroom, they reflected lamplight off the water surface onto a screen, and took video images of the screen (see fig. (1). This screen was shielded from any direct light from the source lamp. The rippled water surface caused intensity variations on the screen, depending on whether the particular perturbation tended to focus or defocus the light, making even very shallow ripples visible (see fig. 5). The intensity pattern then indicated the progress of the ripples from source to image, and back. They then analysed the video by eye, frame by frame, to locate the times and positions of the reformations.

The CT/JT setup used lightproof box to eliminate stray light. Inside the box they imaged the reflection of a diffuse light source directly using a high resolution webcam (see fig. 6). This enabled them to electronically process the images.

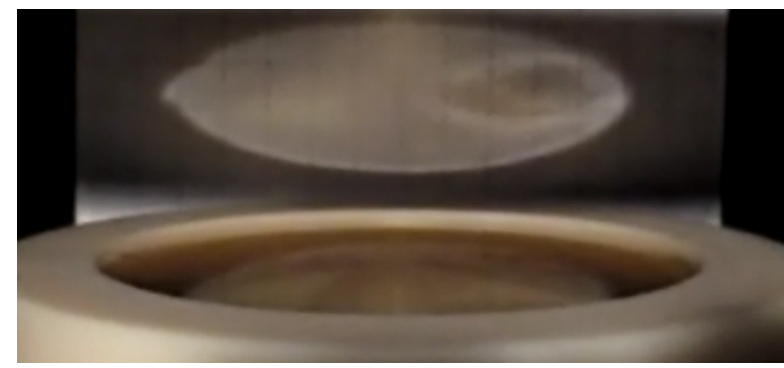

FIG. 5: A snapshot of the NK/TT experiment in progress, with the Fishpond in the foreground, and the screen above and behind. We can clearly see the reflected ripple patten on the right of the screen; this image was taken just before the second reformation.

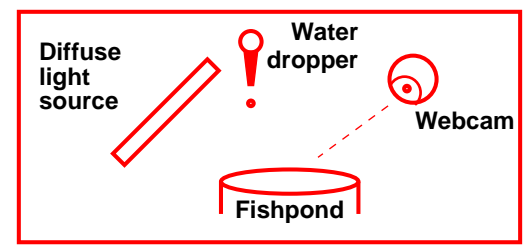

Light-proof box

FIG. 6: The experimental setup of CT and JT. In addition the Fishpond was placed in a waterbath with a heat pump, to enable the temperature to be changed in a controllable way.

In both experiments, ripples were created using a water dropper to drop a single water droplet into the Fishpond; the height of which was varied to ensure that the strongest possible ripples were generated, but not so big as to be accompanied by splashing or bubbles. A full range of starting positions was investigated, since the Fishpond should create reformations from any point although starting positions near the wall suffered due to edge effects.

The positioning of screens, light sources, cameras, and so on were systematically varied to achieve the best images. For NK/TT, a shallow angle of reflection enhanced the images, although if too shallow this significantly reduced the fraction of the water surface that could be seen. For CT/JT, the diffuse light source was placed at a low angle, but with the camera at a $90^{\circ}$ reflectance angle; thus giving a strong contrast from the light reflected off the ripples. The diffuse light source avoided problems caused by reflections off the bottom centre of the Fishpond.

\section{A. Setup 1}

The first experiment, performed by NK and TT, used the setup of fig. 目, where videos were taken from each initial droplet to final dissipation of the ripples. Each instance was viewed carefully frame-by-frame to determine the time taken for the easily detectable 1st and 2nd reformations. 


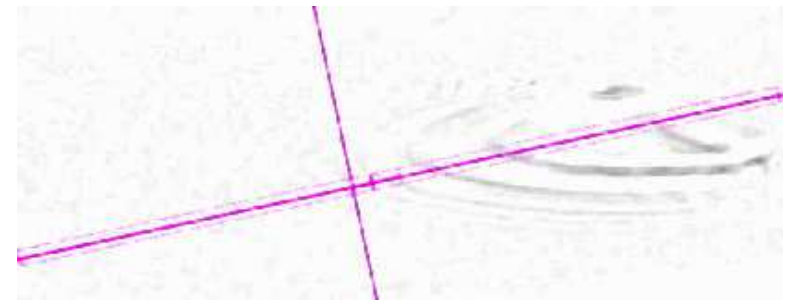

FIG. 7: Typical differential image obtained by CT and JT, after first subtracting one webcam frame from the next, and them removing speckle, in order to enhance ripple visibility .The purple lines cross the centre of the Fishpond, with the more horizontal on a line linking the source-point to the reformation point, with the other at right angles from the centre of the pond. The image is foreshortened due to the angle of view of the webcam.

Reformation times were found by first locating the desired reformation within a short "target" sequence of video frames. With each reformation point taken to be indicated by the frame with the most localised ripple pattern, the short target sequence was viewed independently by NK and TT, both forward and backwards. Once the best reformation frame(s) were chosen, the reformation time could be calculated using the frame rate of the camera. NK and TT also attempted to increase the longevity of the ripples in order to detect third reformations. However, reducing the water viscosity (and hence dissipation) by using water at $70 \mathrm{C}$ had negligible effect, and reducing the surface tension using either detergent or temperature slowed the wave speeds, increasing the effect of dissipation.

The positions of source, and 1st and 2nd reformation points were also compared and measured. However, since the foreshortening of the reflected ripple pattern means that it appears as an ellipse, times were calculated from instances where the source and reformation points appeared on a horizontal line on the screen, i.e. the long axis of the ellipse.

The average times taken for first and second reformations were $(1.04 \pm 0.04) \mathrm{s}$ and $(1.07 \pm 0.12) \mathrm{s}$. The variation between times taken for first reformations of different ripples at varied start positions was dominated by video frame rate. The larger variation for the second reformations was due to the difficulty in determining the video frame with the most localized ripple pattern, since by then the ripples had both dispersed and diminished significantly.

The theoretically expected reformation time can be calculated most easily by referring to the reference sphere. The water depth at the centre of the Fishpond matches that on the imaginary reference sphere, which in our case is $2.5 \mathrm{~mm}$ deep, leading to a wave velocity of $v=\sqrt{g d} \approx 157 \mathrm{~mm} / \mathrm{s}$. Each reformation on the sphere takes place after one half circumnavigation, and the sphere radius $s$ is half that of the Fishpond radius of $r_{0}=100 \mathrm{~mm}$; thus the theoretical reformation time is

$$
T=\frac{\pi s}{v_{0}} \approx \frac{3.142 \cdot 50 \mathrm{~mm}}{157 \mathrm{~mm} / \mathrm{s}} \approx 1.003 \mathrm{~s},
$$

which seems to be in good agreement with the measurements - but see the discussion in section $\mathrm{V}$.

\section{B. Setup 2}

In the second experiment, $\mathrm{CT}$ and JT directly imaged the ripples using a webcam and VirtualDub [26, as shown in fig. 6. To emphasize the ripple dynamics, they subtracted each frame from the previous one using AviSynth 27. The resulting differential image data as shown in fig. 7, reveals only the wave motion, which was then analysed using Tracker [28]. Each image was scanned along the axis between source and image points, giving a $2 \mathrm{D}$ dataset, comprising a time series of $1 \mathrm{D}$ datasets along this axis. The result was processed and plotted using a variety of software, including Microsoft Excel, Matlab 29], and Scilab 30].

In order to optimise the reformation process, $\mathrm{CT}$ and JT systematically analysed results taken for a range of temperatures and fill volumes, as shown in tables [1 and II. The optimum temperature was found to be 15C: although the water viscocity (and loss) increases for lower temperatures, the surface tension increases, leading to faster wave speeds. Note that the optimum fill volume centred around $190 \mathrm{ml}$, this can be compared to that for the design parameters, which radial integration of the design depth profile is found to be $183 \mathrm{ml}$. This is in good agreement - a $3 \mathrm{ml}$ change in fill volume corresponds to about a $0.1 \mathrm{~mm}$ depth change, and velocity shifts of less than $2 \%$. The experimental optimum filling volume of $190 \mathrm{ml}$ is higher than the design volume, perhaps because of the way the Fishpond fills - e.g. the design takes no account of surface tension.

In fig. 8(a) we see the first reformation very clearly, although dispersion has spread out the initial impulse introduce by a falling water drop. On the first traversal, we can not only see the evidence of several ripple crests, but also the slight fanning as the ripples disperse. Further reformations, although apparent to the eye, do not show up over the imaging noise.

\begin{tabular}{|l|c|c|c|c|c|c|c|}
\hline Volume [ml] & 170 & 175 & 180 & 185 & 190 & 195 & 200 \\
\hline Reformations & 2 & 2 & 2 & 3 & 3 & 3 & 2 \\
\hline
\end{tabular}

TABLE I: Relationship between water volume in the Fishpond and the number of reformations visible to the eye.

Reformation times and errors were extracted from data like that shown on fig. 8 using a curve fitting process. For example, at the optimum temperature of $15 \mathrm{C}$, the first reformation was calculated to have occured at the 23rd frame (at $0.72 \pm 0.06 \mathrm{~s}$ ), with the second being 30 frames later $(+1.03 \pm 0.11 \mathrm{~s})$. The second reformation 

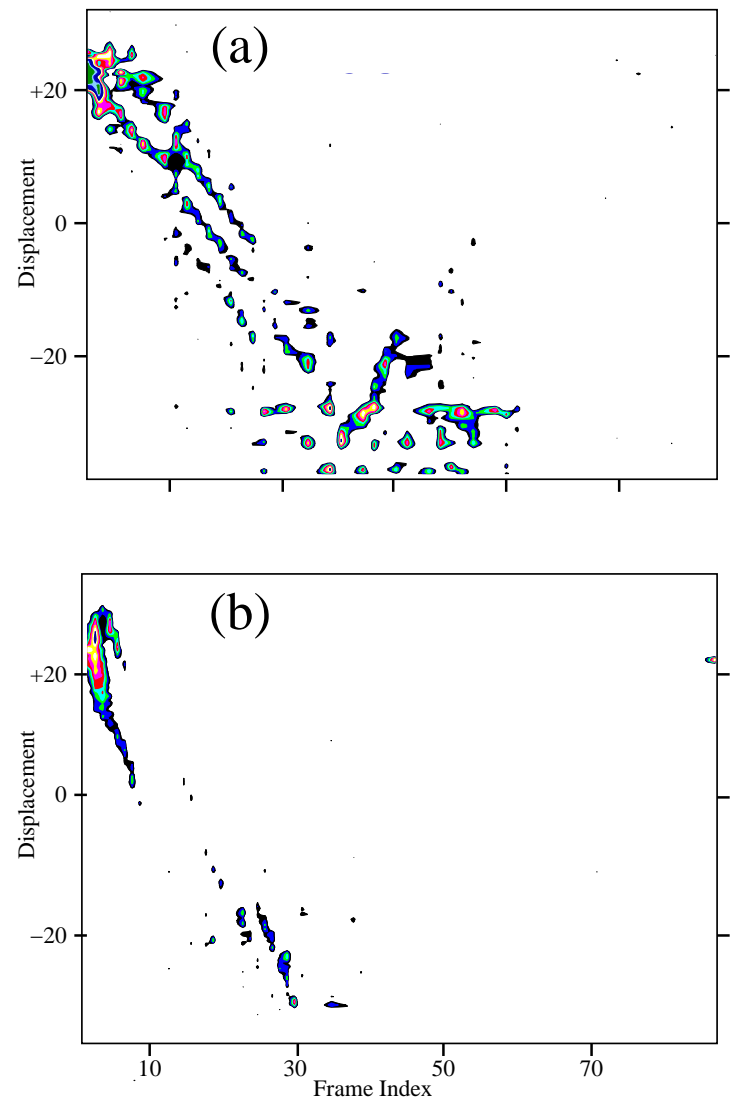

FIG. 8: Differential luminance data indicating the presence of ripples along the axis between initial disturbance and reformation point. As time (and frame index) progresses, we see the ripples travel from source to reformation, although the pattern is complicated by the two possible paths - either with an early reflection off the bowl edge, or with a late reflection. This is in addition to the spreading out of the ripple pattern due to dispersion. Results for two different water temperatures are shown, at both (a) $15 \mathrm{C}$, and (b) $20 \mathrm{C}$, with $15 \mathrm{C}$ water giving better data, in agreement with Table II. These contour plots are made by averaging over adjacent points and using a logarithmic scale; the contours are evenly spaced, with a minimum level chosen to best display the ripple patterns.

\begin{tabular}{|l|c|c|c|c|c|c|c|c|c|}
\hline Temperature [C] & 0 & 5 & 10 & 15 & 20 & 25 & 30 & 35 & 40 \\
\hline Reformations & 1 & 2 & 2 & 3 & 2 & 2 & 2 & 2 & 2 \\
\hline
\end{tabular}

TABLE II: Relationship between temperature of the Fishpond and the number of reformations visible to the eye.

was notably slower than the first, but then the error is also much larger; but of course some slowing might be expected since the longer wavelengths both persist longer and travel more slowly.

Finally, despite the difficulty in extracting second and third reformation times from the video data, and in seeing them in plots such as fig. 8, in the original videos themselves the third reformation is clearly visible to the eye. This suggests that significant performance improvements are still possible in the automated processing of the data.

\section{SURFACE TENSION}

Since water is the obvious liquid to use in the Fishpond, and it has a significant surface tension, we should estimate its effects. The wave velocity including the effects of surface tension $\sigma$ (in $\mathrm{N} / \mathrm{m}$ ) on waves of wavelength $\lambda=2 \pi / k$, in a fluid of density $\rho$ and depth $d$ is

$$
v^{2}=\frac{\omega^{2}}{k^{2}}=\frac{g}{k}\left[1+\frac{\sigma k^{2}}{g \rho}\right] \tanh (k d) .
$$

Thus the correction to the leading term which gives us the shallow water wave speed is a factor $\epsilon=\sigma k^{2} / g \rho$. Surface tension in water reduces with temperature, being about $0.073 \mathrm{~N} / \mathrm{m}$ at $20 \mathrm{C}$, but $0.061 \mathrm{~N} / \mathrm{m}$ at $90 \mathrm{C}$. At about $20 \mathrm{C}$,

$$
\epsilon=\frac{\sigma k^{2}}{g \rho} \approx \frac{0.073 k^{2}}{9.81 \times 1000}=7.44 \times 10^{-6} k^{2} .
$$

For water waves of wavelength $20 \mathrm{~mm}, k=2 \pi / 0.02 \approx$ $314 \mathrm{~m}^{-1}$, so that $\epsilon \approx 0.73$; the wave speed is therefore a factor of $\sqrt{1.73}$ (or 30\%) higher than expected based on depth alone, and is wavelength dependent even in the shallow water limit. However, this speed shift is not depth dependent, so the refocussing character of the Fishpond is unaffacted - but different wavelengths reform at different times. This dispersion means that a determination of the reformation time becomes harder as time progesses, and will depend on the specific details of how a given reformation time is evaluated.

Initially, the NK/TT measured reformation time of $(1.04 \pm 0.04) \mathrm{s}$ seemed in good agreement with the simple prediction of eqn. (10), i.e. 1.00s. However, we can now see that surface tension effects should reduce this prediction by $30 \%$ to about $0.7 \mathrm{~s}$, which agrees with that measured by $\mathrm{CT} / \mathrm{JT}$, and not that of NK/TT.

But why do the two experiments give such different outcomes? Two scenarios, which are not mutually exclusive, suggest themselves.

First, the criteria for choosing the reformation times differed, and this will affect which frame of video selected - NK/TT chose by eye the frame with the smallest region of disturbed water, whereas CT/JT applied a simple fitting algorithm to digitised data along one axis.

Second, NK/TT relied upon the reported frame rate of their camera, and perhaps this was not reliable; although with hindsight we realise that their framerates might have been easily calibrated by videoing a clock either before or at the same time as the each experimental run.

It is gratifying that the more sophisticated setup of $\mathrm{CT} / \mathrm{JT}$ gives good agreement with theory, although it is not clear why the first attempt by NK/TT did less well. Neverthless, one of the features of this student project was it could be implemented in many different ways - the students were given the Fishpond, some reading material, 
and some suggestions and then largely left to get on with it as independently they wished. Still other experimental set-ups and measurements are possible, and so we expect that the Fishpond itself will be reused many times in the future.

\section{SUMMARY}

We have shown how an exotic phenomenon from transformation optics - the Maxwell's fisheye lens - can be converted into simple water waves in a tabletop "Maxwell's Fishpond". This is currently being used sucessfully as a third year undergraduate experimental project in the Physics Department of Imperial College London. While the remarkable series of image reformations provides the hook which makes the project interesting, there are many other features that can be investigated as part of the experiment. Most straightforwardly, there is a variety of imaging possibilities to be investigated (two of which were discussed here), and various experimental conditions - lighting, fill depth, etc - to be determined. Also, the effect of viscosity on performance can be tested by changing the water temperature, or surface tension can be removed by adding detergent or other liquids might be used. A transparent Fishpond might be made so as to image the ripples in transmission, or a vibrating source could be used in an attempt to generate standing waves. For the more mathematically inclined, the nature of stereographic projections can be researched, other comparable devices - e.g. the Eaton or Luneburg lenses - considered, or numerical simulations attempted. Alternatively, rather than only aiming to optimise the number of reformations, or visibility to the eye, but it is also possible to consider ease or simplicity of fabrication, with a view to testing performance as a function of size. Since our simulations show that the general behaviour persists even for an approximate depth profile - such as a shallow dome - sophisticated or precise manufacturing processes are not needed.

In this way, this simple, eye-catching device provides a rich playground in which a wide variety of students can test their skills while investigating a novel device not only part of contemporary research - that of transformation optics and acoustics - but with a history that goes back to Maxwell himself.

\section{Appendix}

\section{A. Other Lenses}

One might also construct Eaton and Luneburg lenses[2], or even their generalizations [1, 22, using water waves. The refractive index profiles, which are proportional to the inverse of the velocity profiles for the Eaton and Luneburg lenses, are $n_{\text {Eaton }}(r)=\sqrt{\left(2 r_{0}-r\right) / r}$ and
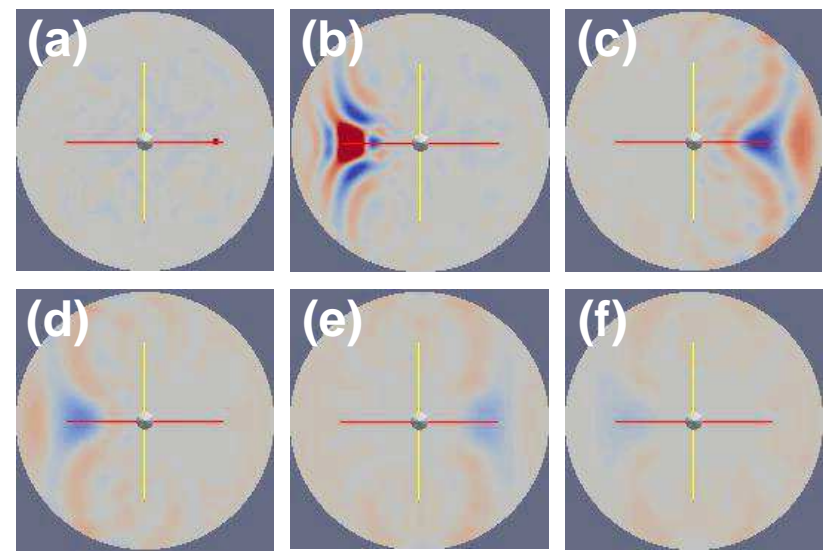

FIG. 9: Snapshots from a Maxwell's Fishpond 200mm diameter and $10 \mathrm{~mm}$ deep, simulated using OpenFOAM, at the start and each subsequent reformation up to the fifth. Although the simulation retains to a reasonable extent the repeated refocussing, the wave dispersion continually increases the length of the pulse of ripples, so that reformations, while still remaining on the scale of a wavelength, have an ever increasing duration.

$n_{\text {Luneburg }}(r)=\sqrt{\left(2 r_{0}^{2}-r^{2}\right) / r_{0}^{2}}$. Then, by comparing velocity profiles between the optical and water wave cases, and choosing a reference depth $d_{0}$ and reference radius $r_{0}$, we find that the retro-reflecting Eaton pond feature and the focussing Luneburg pond feature need the depth profiles

$$
\begin{aligned}
d_{\text {Eaton }}(r) & =\frac{r d_{0}}{2 r_{0}-r}, \\
d_{\text {Luneburg }}(r) & =\frac{r_{0}^{2} d_{0}}{2 r_{0}^{2}-r^{2}} .
\end{aligned}
$$

\section{B. Other waves}

It is possible to imagine other implementations of the Maxwell fisheye concept. For example, an adaption of the expertise demonstrated by Bramhavar et al in 23. might give rise to an appropriately tapered "Maxwell's Platter" with the same behaviour for acoustic waves in a solid.

\section{Finite Element simulations}

As an estimator of the necesarily imperfect fishpond experiment, we (PK) also did more realistic finite element simulations using the open source simulator OpenFOAM 25] using the interMixingFoam engine, on a fast desktop PC. Despite computational constraints, the general character of the idealised process was preserved, and the effect of dispersion demonstrated. A typical simulation result is shown on fig. 9; others indicate that larger fishponds may perform better than ours - although will be harder to construct, and will suffer more from dispersion. 


\section{MEEP ctl files}

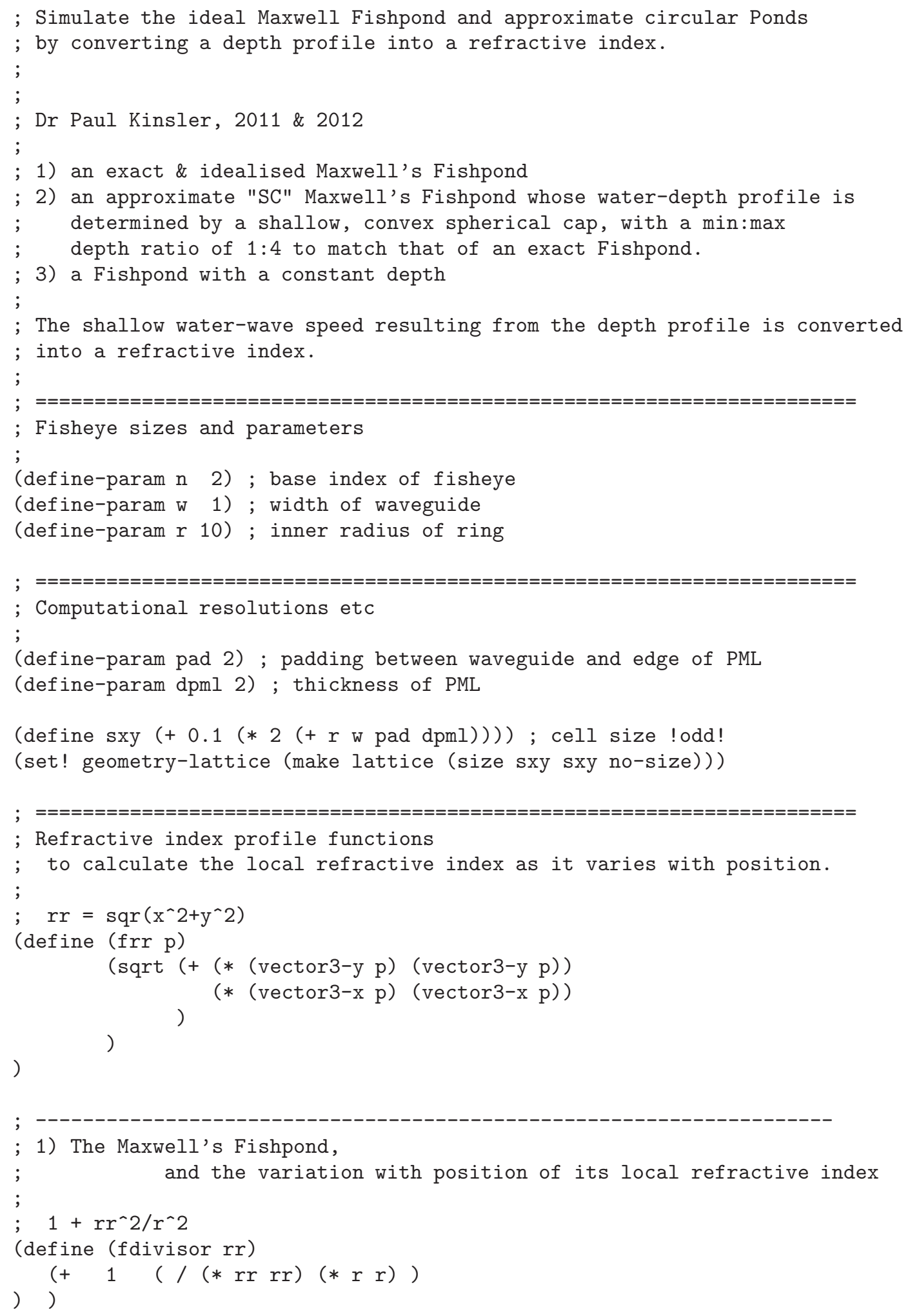




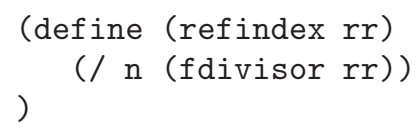


[1] T. Tyc, L. Herzánová, M. Sarbort, and K. Bering, New J. Phys. 13, 115004 (2011), doi:10.1088/1367-2630/13/11/115004.

[2] R. K. Luneburg, Mathematical Theory of Optics (University of California Press, Berkeley, CA, 1964).

[3] Y. G. Ma, S. Sahebdivan, C. K. Ong, T. Tyc, and U. Leonhardt,

New J. Phys. 13, 033016 (2011), doi:10.1088/1367-2630/13/3/033016.

[4] A. V. Kildishev and V. M. Shalaev, Phys.-Usp. 54, 53 (2011), URL http://iopscience.iop.org/1063-7869 54/1/R05.

[5] H. Chen and C. T. Chan, J. Phys. D 43, 113001 (2010), URL http://iopscience.iop.org/0022-3727 43/11/1130017.

[6] M. W. McCall, A. Favaro, P. Kinsler, and A. Boardman. J. Opt. 13, 024003 (2011), URL http://iopscience. iop.org/2040-8986/13/2/024003.

[7] The Cambridge and Dublin Math. Journal 8, 188 (1853).

[8] The Cambridge and Dublin Math. Journal 9, 9 (1854).

[9] U. Leonhardt, New J. Phys. 11, 093040 (2009), doi:10.1088/1367-2630/11/9/093040

[10] R. J. Blaikie, New J. Phys. 13, 125006 (2011), doi:10.1088/1367-2630/13/12/125006.

[11] P. Kinsler and A. Favaro, New J. Phys. 13, 028001 (2011), doi:10.1088/1367-2630/13/2/028001; arXiv:1010.2346

[12] T. Zentgraf, Y. Liu, M. H. Mikkelsen, J. Valentine, X. Zhang. Nat. Nano. 6, 151 (2011), doi:10.1038/nnano.2010.282.

[13] M. Kadic, G. Dupont, S. Guenneau, and S. Enoch, J. Mod. Opt. 58, 994 (2011), doi:10.1080/09500340.2011.5899

[14] G. C. Righini, V. Russo, S. Sattini, and G. T. D. Francia, Appl. Opt. 11, 1442 (1972), doi:10.1364/AO.11.001442.

[15] V. N. Smolyaninova, I. I. Smolyaninov, A. V. Kildishev, V. M. Shalaev,

Opt. Lett. 25, 3396 (2010) doi:10.1364/OL.35.003396.
[16] L. H. Gabrielli. U. Leonhardt, and M. Lipson (2010), arXiv:1007.2564. Although now sadly withdrawn by the authors, this attempt to build a Maxwell's fisheye device is nevertheless worthy of note, whatever the final outcome.

[17] L. H. Gabrielli M. Lipson,

J. Opt. 13, 024010 (2011).

doi:10.1088/2040-8978/13/2/024010

[18] G. Jannes, R. Piquet, J. Chaline, P. Maïssa, C. Mathis, G. Rousseaux, J. Phys: Conf. Ser. 314, 012031 (2011), doi:10.1088/1742-6596/314/1/012031.

[19] G. Rousseaux, C. Mathis, P. Maïssa, T. G. Philbin, U. Leonhardt, New J. Phys. 10, 053015 (2008), doi:10.1088/1367-2630/10/5/053015.

[20] T. Foglizzo, F. Masset, J. Guilet, and G. Durand, Phys. Rev. Lett. 108, 051103 (2012), doi:10.1103/PhysRevLett.108.051103.

[21] N. Mayo, The Physics Teacher 35, 352 (1997).

[22] M. Šarbort and T. Tyc, J. Opt. 14, 075705 (2012).

[23] S. Bramhavar, C. Prada, A. A. Maznev, A. G. Every, T. B. Norris, T. W. Murray, Phys. Rev. B 83, 014106 (2011), doi:10.1103/PhysRevB.83.014106.

[24] A. F. Oskooi, D. Roundy, M. Ibanescu, P. Bermel, J. D. Joannopoulos, S. G. Johnson,

Comput. Phys. Commun. 181, 687 (2010), doi:10.1016/j.cpc.2009.11.008.

[25] OpenFOAM. The OpenFOAM Foundation, URL http://www .openfoam .org/.

[26] VirtualDub, URL http://www.virtualdub.org/

[27] AviSynth,URL http://avisynth.org/.

[28] D. Brown. Tracker. URL http://www . cabrillo.edu/dbrown/tracker/.

[29] MatLab, MathWorks URL http://www . mathworks . com/products/matlab.

[30] Scilab, Scilab Consortium URL http://www.scilab.org/. 